\title{
Bone mineral density in acromegaly: the effect of gender, disease activity and gonadal status
}

\begin{abstract}
Alfredo Scillitani ${ }^{*}$, Claudia Battista*, lacopo Chiodini $^{\star \star \star \star *}$, Vincenzo Carnevale*, Saverio Fusilli*, Enrica Ciccarellit, Massimo Terzoloł, Giuseppe Oppizzi§, Maura Arosioๆ, Maurizio Gasperi**, Giorgio Arnalditt, Annamaria Colaoł‡, Roberto Baldelli§§, Maria Rosaria Ghiggi*`ๆn, Daniela Gaia†, Carolina Di Sommatt, Vincenzo Trischitta* and Antonio Liuzzitt† *Ospedale 'Casa Sollievo della Sofferenza' IRCCS, San Giovanni Rotondo (FG), †Department of Internal Medicine, Division of Endocrinology, University of Torino and $\ddagger$ Department of Clinical and Biological Sciences, $1^{\text {st }}$ Internal Medicine, A. O. San Luigi, 10043 Orbassano, Turin, $\S$ Department of Endocrinology, Niguarda Hospital and IInstitute of Endocrine Sciences, University of Milano, Ospedale Maggiore IRCCS, Milan, ${ }^{* *}$ Department of Endocrinology, University of Pisa, Pisa, ††Division of Endocrinology, Department of Internal Medicine, University of Ancona, Ancona, $\ddagger \ddagger D e p a r t m e n t$ of Molecular and Clinical Endocrinology and Oncology, Federico II University, Naples, §§Department of Clinical Science, Endocrine Section, University of Roma 'La Sapienza', Rome, १†Division of Internal Medicine, Busto Arsizio and ${ }^{* \star \star}$ Unit of Endocrinology, Ospedale 'San GiuseppeFatebenefratelli', A.Fa.R. Milan, and †††Division of Endocrinology and Metabolic Diseases, Istituto Auxologico Italiano, Ospedale San Giuseppe, Verbania, Italy

(Received 2 September 2002; returned for revision 14 October 2002; finally revised 3 November 2002; accepted 22 January 2003)
\end{abstract}

\section{Summary}

OBJECTIVE Data on bone mineral density (BMD) in acromegaly are conflicting as most previous studies collectively evaluated eugonadal and hypogonadal patients of both sexes, with or without active disease. We have evaluated BMD in 152 acromegalic patients of both sexes with varying disease activity and gonadal status.

DESIGN Cross-sectional, retrospective.

Correspondence: Alfredo Scillitani, Division and Research Unit of Endocrinology, Ospedale 'Casa Sollievo della Sofferenza', IRCCS, 71013 San Giovanni Rotondo (FG), Italy. Tel.: +39 0882410625; Fax: +39 0882451637. E-mail: alscill@tin.it
PATIENTS We studied 152 acromegalic patients (99 women aged 26-72 years, and 53 men aged 2175 years), 107 with active and 45 with controlled disease. Eighty-five patients had normal gonadal status and 67 were hypogonadal.

MEASUREMENTS In all patients we measured serum GH levels by immunoenzimometric assay, and serum IGF-I levels by radioimmunoassay. BMD was assessed at spine L2-L4 (LS) and at femoral neck (FN) by dual energy X-ray absorptiometry; results are expressed as Z-values.

RESULTS We evaluated the effect of $\mathrm{GH}$ excess on bone at different sites in relation to gonadal status, disease activity and gender. At LS, in respect to the reference population, BMD (mean $\pm \mathrm{SE}$ ) values were higher in eugonadal patients (active: $0.71 \pm 0.29$, $P<0.02$; controlled: $0.65 \pm 0.28, P<0.05)$ and lower in hypogonadal ones (active: $-0.64 \pm 0.35,0.1<P<0.05$; controlled: $-1.05 \pm 0.36, P<0.01$ ), regardless of disease activity. On the contrary, at FN, BMD was higher than in the reference population, both in eugonadal $(1.01 \pm 0.22, P<0.001)$ and hypogonadal $(0.63 \pm 0.17$, $P<0.001)$ patients only in subjects with active disease, but not in those in which the disease was controlled (eugonadal: $0.31 \pm 0.23, P=n s$; hypogonadal $0.04 \pm 0.28, P=n s)$. We did not observe any difference in BMD values according to gender both at LS (males vs. females $-0.02 \pm 0.30$ vs. $0.01 \pm 0.24, P=n s$ ) or at FN $(0.77 \pm 0.19$ vs. $0.63 \pm 0.15, P=n s)$.

CONCLUSIONS The anabolic effect of $G H$ excess on bone in acromegalic patients is: (i) gender-independent; (ii) evident at the spine only in eugonadal regardless of disease activity; (iii) evident at femoral neck only in the presence of active disease regardless of gonadal status.

It has been known for over 50 years that acromegaly affects bone (Albright \& Reifeinstein, 1948). However, data on bone mineral density (BMD) in these patients are still conflicting. Seeman et al. (1982) detected increased spinal BMD in seven patients with active acromegaly. Diamond et al. (1989) observed reduced spinal BMD in 12 hypogonadal patients but 12 eugonadal patients did not show any difference with respect to controls at 
the spine, while forearm BMD was increased in patients with active disease. Ezzat et al. (1993) showed that spinal BMD was not different from controls in seven eugonadal patients with active disease, while it was reduced in seven hypogonadal patients with active disease. Ho et al. (1992) observed 25 patients with active disease, eight eugondal and 17 hypogonadal subjects, and did not observe any difference at spine and femur BMD with respect to controls. Kotzman et al. (1993) examined 16 patients with active disease, four eugonadal and 12 hypogonadal, and detected increased BMD at spine and femur. Kayath \& Vieira (1997) studied 45 patients (11 eugonadal and 34 hypogonadal subjects) with active disease and did not report changes in BMD measured at spine and femur. Lesse et al. (1998) studied 18 patients ( 10 with active disease and 12 hypogonadal subjects) and analysed data in terms of activity of disease and gonadal status, in eugonadal patients detected higher BMD at spine and femur, and in hypogonadal patients reduced spinal BMD. Longobardi et al. (1998) studied 11 patients with active disease (six eugonadal and five hypogonadal) and demonstrated a reduced spinal BMD. Kaji et al. (2001) examined 26 patients with active disease, and found higher BMD at the spine, femur and forearm areas compared to controls. There are several reasons for these conflicting results: the small sample size of previous studies, and in some of these studies the lack of patient stratification and separate analysis according to gender, disease activity and gonadal status. Moreover, BMD was measured at different skeletal sites, whose behaviour with respect to GH excess and hypo- or eugonadal status could be divergent and therefore not comparable (Diamond et al., 1989; Ho et al., 1992; Kotzman et al., 1993; Kayath \& Vieira, 1997; Lesse et al., 1998; Longobardi et al., 1998). To investigate the skeletal involvement in acromegalic patients, and to evaluate the effect of the aforementioned factors, a large sample of patients is needed. Therefore, we retrospectively evaluated BMD both at spine and at femoral neck in over 150 acromegalic patients, enrolled in nine referral Italian centres, which participated in the Italian Study Group of Acromegaly under the auspices of the Italian Society of Endocrinology (SIE).

\section{Patients and methods}

\section{Patients}

One hundred and seventy-three acromegalic patients, referred to nine centres in Italy, were initially studied. In all patients, the diagnosis of acromegaly had been suspected by clinical features and confirmed by high serum GH levels not suppressible below $1 \mu \mathrm{g} / 1$ after oral glucose tolerance test (OGTT) and high serum IGF-I level compared to gender and age-related normal values.

At the time of observation, 41 patients had been diagnosed recently. The remaining 132 patients had been previously submitted to one or more of the following treatments: surgery (90), radiotherapy (40), or pharmacological therapy with long-acting agonists of somatostatin or dopamine agonists (104). Twenty-one patients were excluded from the study because they were either affected by concomitant diseases (six by thyrotoxicosis, three by breast cancer, one by lymphoma and one by progressive systemic sclerosis) or they had been treated with therapeutic agents (six alendronate and four high-dose corticosteroids) known to affect bone metabolism and influence BMD.

Therefore we finally studied 152 patients: 99 women (aged 26 72 years, mean \pm SE $50 \cdot 8 \pm 1 \cdot 13$ years) and 53 men (aged $21-$ 75 years, mean \pm SE $48 \cdot 1 \pm 1 \cdot 75$ years). According to IGF-I serum levels, patients were subdivided in 'active' and 'controlled' groups on the basis of specific gender- and age-adjusted reference range (Giustina et al., 2000). At the time of observation, serum IGF-I levels were within the normal range according to gender and age in 45 patients ( 33 women, 12 men); in 23 of these patients, disease activity had been controlled by surgery and/or radiotherapy and in 22 by pharmacological therapy. However, among these 45 patients, 15 had mean serum GH levels higher than $2.5 \mu \mathrm{g} / 1$, which is considered the upper limit for an adequate control of acromegaly (Giustina et al., 2000). Serum IGF-I levels were higher than normal in 107 patients. The duration of disease was estimated by interviewing the patients about the time of onset of acral enlargement and by watching patient's photographs over the previous decades. The estimated time of onset of acromegaly was after 19 years of age for all patients.

Eighty-five patients (66 females and 19 males) were eugonadal, whereas 67 patients were hypogonadal (33 females and 34 males). The duration of hypogonadism was estimated by interviewing all patients about symptoms, taking into account their menstrual history and serum oestradiol levels (oestradiol $<110 \cdot 1 \mathrm{pmol} / \mathrm{l}$ ) in females, and serum testosterone levels (testosterone $<8.67 \mathrm{nmol} / \mathrm{l}$; Anonymous, 2001) in males. Length of hypogonadism in female patients, who were not on sex steroid replacement therapy, was arbitrarily defined as lasting from the time of onset of symptoms to the age of 50 years, which is the mean menopausal age of Italian women (Parazzini et al., 1992). At the time of observation, patients on sex steroid replacement therapy (eight females and eight males with active disease, seven females and five males with controlled disease) had been treated for at least 5 years. Thirty patients were on substitutive glucocorticoid and/or thyroid hormone therapy. None of the patients had renal or hepatic impairment and none consumed substances known to affect bone metabolism. All patients gave their witnessed informed consent before entering the study, whose design was made according to the Helsinki Declaration II.

\section{Patients' classifications}

According to the above characteristics, patients were analysed either as a whole group or in different subgroups according to 
disease activity (active/controlled) or gender (male/female). Within these groups, patients were also further subdivided according to gonadal status (eugonadism, hypogonadism).

\section{Measurements}

In all patients, a general biochemical profile of the serum plus evaluation of thyroid function was performed to exclude diseases influencing bone metabolism. Serum GH levels were measured by immunoenzymometric assay (Eurogenetic Italy, Turin, Italy); intra- and interassay CVs were $3 \cdot 3-2 \cdot 6-2 \cdot 7 \%$ and $4 \cdot 2-5 \cdot 4-4 \cdot 3 \%$, respectively, at the mean concentration of $2 \cdot 8-23 \cdot 6-42 \cdot 2 \mu \mathrm{g} / 1$. Serum IGF1 levels were evaluated by radioimmunoassay (RIA) after acid-ethanol extraction (Nichols Institute Diagnostics, BT Wychen, the Netherlands); intra- and interassay CVs (IGF1) were $2 \cdot 4-3 \cdot 0-3 \cdot 6 \%$ and $5 \cdot 2-8 \cdot 4-6 \cdot 8 \%$, respectively, at the mean concentration of $119 \cdot 2-207-540 \mu \mathrm{g} / 1$.

In all patients, BMD was measured at spine (L2-L4) and/or at femoral neck by dual X-ray absorptiometry (DXA) using Hologic (110 patients, 66 with active disease and 44 with controlled disease; Hologic Inc, Waltham, MA, USA) or Norland (42 patients, 39 with active disease and three with controlled disease; Norland Instruments, Fort Atkinson, WI, USA) densitometers, whose in vitro mean precision at spine and femoral neck was $1 \cdot 0$ and $2 \cdot 3 \%$, respectively. BMD was not measured at lumbar spine (LS, L2-L4) in 24 patients, and at femoral neck (FN) in 17 patients because of arthropathic involvement at spine or hip, vascular calcifications or spinal fractures evidenced by X-ray of lumbar spine and hip.

In order to evaluate volumetric trabecular bone density in 40 patients (23 females and 17 males) with active disease, spinal trabecular BMD was measured also by single-energy quantitative computed tomography L1-L4 (QCT; Toshiba CT Xpeed; Toshiba Medical Systems Division, Tokyo, Japan; in vivo precision $1 \cdot 8 \%$ ).

In order to express BMD data with respect to the different ages and gender of patients and to the different devices, BMD data were expressed as SD units (or Z-transformed) by relating them to the reference population of each centre (Scillitani et al., 1997). Bone mass in the reference population of each centre was comparable to each of the others (Guglielmi et al., 1995).

\section{Statistical analyses}

Results are expressed as mean \pm SE. Significant difference was assumed at $P<0 \cdot 05$. Significant differences in BMD between patients and reference population were assumed when BMD Zvalues divided by SE were greater than $t$-value according to the sample size (Lesse et al., 1998).

The comparison between patients with active and controlled disease, and that between women and men, was performed using unpaired $t$-test or Mann-Whitney $U$-test, as appropriate. Categorical variables were analysed by $\chi^{2}$ test.

In order to compare data of different subgroups stratified by disease activity or sex and gonadal status (i.e. eugonadal and hypogonadal patients of different sex; eugonadal and hypogonadal patients with active or controlled disease) one-way analysis of variance (ANOVA) and Bonferroni post hoc analysis were performed.

In order to evaluate at LS the correlation between volumetric BMD measured by QCT and areal BMD measured by DXA, Spearman rank order correlation was performed.

Multiple regression analysis was performed considering BMD as dependent variable, and IGF-I levels, 'duration of acromegaly' and 'duration of hypogonadism' as independent variables.

\section{Results}

\section{Relationship between BMD and disease activity}

The characteristics of active or controlled patients are shown in Table 1. Except for GH levels, age, BMI, disease duration, proportion of eugonadal and hypogonadal subjects and duration of hypogonadism were similar in active and controlled patients.

Table 1 Clinical characteristics of the patients studied according to disease activity

\begin{tabular}{lllr}
\hline & Active & Controlled & $P$-value \\
\hline IGF-I $(\mu \mathrm{g} / \mathrm{l})$ & $800 \pm 33(348-1976)$ & $218 \pm 17(24-466)$ & $<0 \cdot 0001$ \\
Sample size $(n)$ & 107 & 45 & $50 \cdot 6 \pm 1 \cdot 8(29-72)$ \\
Age $($ years $)$ & $49 \cdot 5 \pm 1 \cdot 1(21-75)$ & $27 \cdot 4 \pm 0 \cdot 8(20-40 \cdot 7)$ & $0 \cdot 66$ \\
BMI $\left(\mathrm{kg} / \mathrm{m}^{2}\right)$ & $28 \cdot 3 \pm 0 \cdot 5(20 \cdot 8-41 \cdot 1)$ & $11 \cdot 2 \pm 1 \cdot 2(2-35)$ & $0 \cdot 13$ \\
Duration of disease (years) & $11 \cdot 7 \pm 0 \cdot 8(1-40)$ & $2 \cdot 3 \pm 0 \cdot 3(0 \cdot 2-10 \cdot 3)$ & $0 \cdot 77$ \\
GH $(\mu \mathrm{g} / \mathrm{l})$ & $23 \cdot 7 \pm 3 \cdot 0(1 \cdot 8-374)$ & $25 / 20$ & $0 \cdot 0001$ \\
Eugonadal/Hypogonadal & $60 / 47$ & $7 \cdot 6 \pm 1 \cdot 5(1-23)$ & $0 \cdot 90$ \\
Duration of hypogonadism (years) & $5 \cdot 8 \pm 0 \cdot 6(1-20)$ & $0 \cdot 68$ \\
\hline
\end{tabular}

Data are expressed as mean \pm SEM (range in parentheses). 
Table 2 Bone mineral density (BMD) measured at lumbar spine (LS) and femoral neck (FN) of active or controlled acromegalic patients with different gonadal status

\begin{tabular}{|c|c|c|c|c|c|c|}
\hline & \multicolumn{3}{|l|}{ LS } & \multicolumn{3}{|l|}{$\mathrm{FN}$} \\
\hline & $n$ & BMD & $P$-value & $n$ & BMD & $P$-value \\
\hline Whole sample & 128 & $-0 \cdot 03 \pm 0 \cdot 19$ & ns & 135 & $0 \cdot 68 \pm 0 \cdot 12$ & $<0.001$ \\
\hline Eugonadal & 66 & $0 \cdot 70 \pm 0 \cdot 23 \S$ & $<0.01$ & 76 & $0 \cdot 82 \pm 0 \cdot 17$ & $<0.001$ \\
\hline Hypogonadal & 62 & $-0 \cdot 75 \pm 0 \cdot 27$ & $<0.01$ & 59 & $0 \cdot 50 \pm 0 \cdot 15$ & $<0.002$ \\
\hline Active disease & 95 & $0 \cdot 07 \pm 0 \cdot 24$ & $\mathrm{~ns}$ & 101 & $0 \cdot 84 \pm 0 \cdot 14^{\wedge}$ & $<0.001$ \\
\hline Eugonadal & 50 & $0 \cdot 71 \pm 0 \cdot 29 *$ & $<0.05$ & 55 & $1 \cdot 01 \pm 0 \cdot 22$ & $<0.001$ \\
\hline Hypogonadal & 45 & $-0 \cdot 64 \pm 0 \cdot 35$ & $0.1<P<0.05$ & 46 & $0 \cdot 63 \pm 0 \cdot 17$ & $<0.001$ \\
\hline Controlled disease & 33 & $-0 \cdot 23 \pm 0 \cdot 27$ & ns & 34 & $0 \cdot 21 \pm 0 \cdot 18$ & ns \\
\hline Eugonadal & 16 & $0.65 \pm 0 \cdot 28^{*}$ & $<0.05$ & 21 & $0 \cdot 31 \pm 0 \cdot 23$ & ns \\
\hline Hypogonadal & 17 & $-1 \cdot 05 \pm 0 \cdot 36$ & $<0.01$ & 13 & $0 \cdot 04 \pm 0 \cdot 28$ & ns \\
\hline
\end{tabular}

Data are expressed as mean \pm SE. $n=$ sample size. BMD values are expressed in terms of $Z$-values. $P$ are referred to comparisons with reference population. ${ }^{\wedge} P=0.018 v s$. patients with controlled disease. $\S P<0.001 v s$. hypogonadal patients. One-way ANOvA was performed to compare the BMDs of eugonadal and hypogonadal patients with active and controlled disease measured at the two skeletal sites: $* P<0 \cdot 05$ vs. hypogonadal patients of the same group.

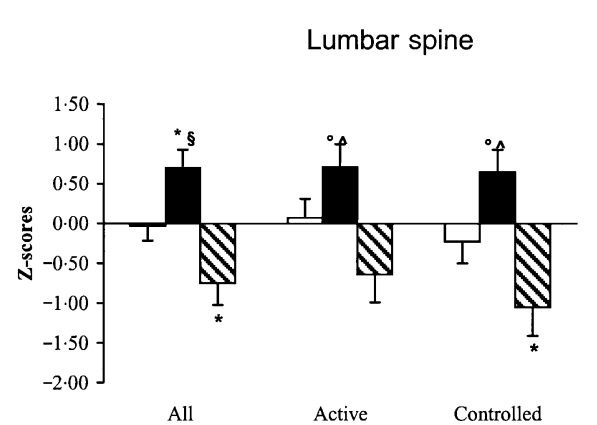

Fig. 1 BMD measured at lumbar spine in active and controlled acromegalic patients with different gonadal status. Data are expressed as Mean \pm SEM. ${ }^{\circ} P<0.05 v s$. reference population; ${ }^{*} P<0 \cdot 01 v s$. reference population. $\S P<0.001 v s$. hypogonadal patients. One-way ANOVA: ${ }^{\wedge} P<0.05 v s$. hypogonadal patients of the same group.

Corticosteroid or thyroxine dosages and percentage of patients consuming these drugs did not significantly differ between subjects with active and controlled disease (data not shown).

As shown in Fig. 1 and Table 2, BMD at LS was higher in eugonadal and lower in hypogonadal patients than in the reference population, regardless of disease activity. Moreover, a significant difference between eugonadal and hypogonadal patients was found both in the active and controlled groups.

As shown in Fig. 2 and Table 2, BMD at FN was significantly higher in the whole sample and in subjects with active disease than in the reference population, independent of gonadal status. In contrast, BMD at FN did not differ from the reference population in patients with controlled disease. Furthermore, BMD at FN was higher in patients with active disease than in patients with controlled disease $(P=0 \cdot 018)$.

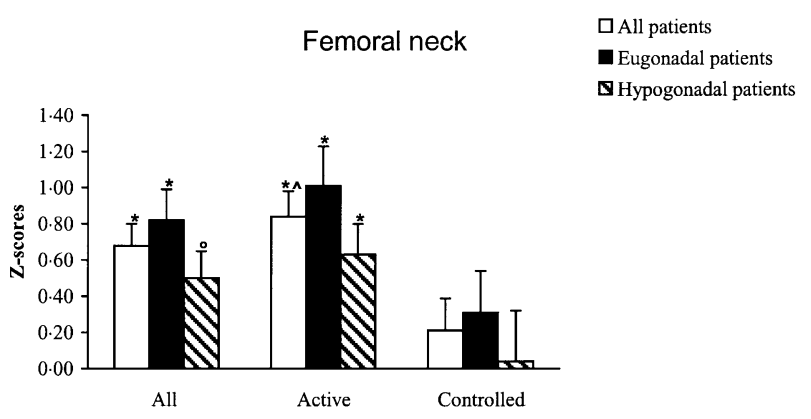

Fig. 2 BMD measured at femoral neck in active and controlled acromegalic patients with different gonadal status. Data are expressed as Mean \pm SEM. ${ }^{\circ} P<0.002 v s$. reference population; ${ }^{*} P<0.001 v s$. reference population. ${ }^{\wedge} P=0.018 v s$. patients with controlled disease.

By restricting the analysis to the 111 patients who measured BMD at both LS and FN, we observed similar results (data not shown). When subdividing the sample of 'controlled' patients on the basis of GH levels (above or below $2 \cdot 50 \mu \mathrm{g} / 1$ ) we did not observe any difference between these subgroups regarding BMD values (Table 3). Moreover, we performed a subanalysis in controlled patients not taking somatostatin analogues, as these drugs could affect bone (Table 4). The results were comparable to those obtained in the all group of controlled patients.

\section{Relationship between BMD and gender}

Male and female patients did not differ for age, BMI, duration of disease, serum GH and IGF-I levels, proportion of active and controlled subjects and duration of hypogonadism, while hypogonadism was more common in males (Table 5). 
Table 3 Bone mineral density (BMD) measured at lumbar spine (LS) and femoral neck (FN) of controlled acromegalic patients with different GH levels (above or below $2.5 \mu \mathrm{g} / 1$ ) and different gonadal status

\begin{tabular}{|c|c|c|c|c|}
\hline & \multicolumn{2}{|l|}{ LS } & \multicolumn{2}{|c|}{ FN } \\
\hline & $n$ & BMD & $n$ & BMD \\
\hline \multicolumn{5}{|l|}{$\mathrm{GH}>2.5 \mu \mathrm{g} / 1$} \\
\hline Eugonadal & 7 & $0 \cdot 36 \pm 0 \cdot 29$ & 9 & $0 \cdot 11 \pm 0 \cdot 42$ \\
\hline Hypogonadal & 5 & $-0.83 \pm 0.66$ & 3 & $-0 \cdot 12 \pm 0 \cdot 85$ \\
\hline \multicolumn{5}{|l|}{$\mathrm{GH}<2.5 \mu \mathrm{g} / 1$} \\
\hline Eugonadal & 9 & $0.87 \pm 0.45^{*}$ & 12 & $0 \cdot 47 \pm 0 \cdot 26$ \\
\hline Hypogonadal & 12 & $-1 \cdot 13 \pm 0 \cdot 44$ & 10 & $0 \cdot 08 \pm 0 \cdot 27$ \\
\hline
\end{tabular}

Data are expressed as mean \pm SE. $n=$ sample size. BMD values are expressed in terms of Z-values. One-way ANOvA was performed to compare the BMDs of eugonadal and hypogonadal patients with controlled disease and GH levels above or below $2.5 \mu \mathrm{g} / 1$, measured at the two skeletal sites. $=P<0.05 v \mathrm{v}$. hypogonadal patients of the same group.

As shown in Fig. 3, the effect of GH excess on BMD measured at LS and FN was not influenced by gender.

\section{Correlations}

Bone mass at LS performed by QCT highly directly correlated with areal BMD measured by DXA $(r=0 \cdot 75, P<0 \cdot 001)$.

\section{Bone mass according to gender}

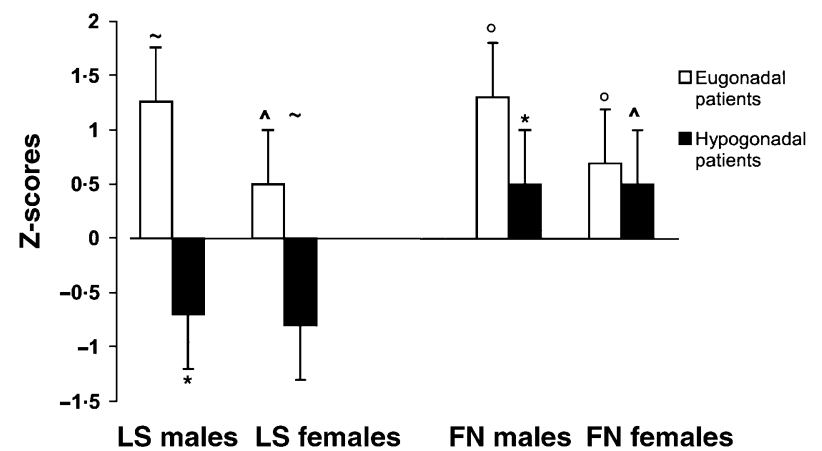

Fig. 3 BMD measured according to gender at lumbar spine (LS) and femoral neck $(\mathrm{FN})$ in acromegalic patients with different gonadal status. Data are expressed as Mean \pm SEM. ${ }^{\wedge} P<0.05 v s$. reference population; ${ }^{*} P<0.01 v$ s. reference population; ${ }^{\circ} P<0.001 v s$. reference population. One-way ANOVA: $\sim P<0.05 v s$. BMD measured at spine in hypogonadal patients of the same gender.

Multiple regression analysis showed that BMD at LS was inversely correlated with 'duration of hypogonadism' $(r=-0 \cdot 30, P<0 \cdot 001$; $\left.R^{2}=0.07\right)$ and positively correlated with 'duration of acromegaly' $\left(r=0 \cdot 23, P<0 \cdot 01 ; R^{2}=0 \cdot 05\right)$, but not with IGF-I levels.

Multiple regression analysis showed that BMD at FN was positively correlated with IGF-I levels $\left(r=0 \cdot 26, P<0 \cdot 01 ; R^{2}=0 \cdot 06\right)$ but not with 'duration of hypogonadism' or 'duration of acromegaly'.

Table 4 Bone mineral density (BMD) measured at lumbar spine (LS) and femoral neck (FN) of controlled acromegalic patients with different gonadal status, not taking somatostatin analogues

\begin{tabular}{|c|c|c|c|c|c|c|}
\hline & \multicolumn{3}{|l|}{ LS } & \multicolumn{3}{|c|}{$\mathrm{FN}$} \\
\hline & $n$ & BMD & $P$-value & $n$ & BMD & $P$-value \\
\hline \multicolumn{7}{|c|}{ Controlled disease } \\
\hline Eugonadal & 5 & $1 \cdot 18 \pm 0 \cdot 47$ & $0 \cdot 1<P<0.05$ & 6 & $0 \cdot 46 \pm 0 \cdot 27$ & ns \\
\hline Hypogonadal & 10 & $-0.99 \pm 0.52$ & $0.1<P<0.05$ & 8 & $-0.15 \pm 0.43$ & ns \\
\hline
\end{tabular}

Data are expressed as mean \pm SE. $n=$ sample size. BMD values are expressed in terms of $\mathrm{Z}$-values. $P$ are referred to comparisons with reference population.

Table 5 Clinical characteristics of the patients studied according to gender

\begin{tabular}{llll}
\hline & Males $(n=53)$ & Females $(n=99)$ & $P$-value \\
\hline Age $($ years $)$ & $48 \cdot 1 \pm 1 \cdot 7(21-75)$ & $50 \cdot 8 \pm 1 \cdot 1(26-72)$ & $0 \cdot 18$ \\
BMI $\left(\mathrm{kg} / \mathrm{m}^{2}\right)$ & $28 \cdot 3 \pm 0 \cdot 5(22-40 \cdot 7)$ & $27 \cdot 8 \pm 0 \cdot 5(20-41 \cdot 1)$ & $0 \cdot 18$ \\
Duration of disease (years) & $11 \cdot 1 \pm 1 \cdot 0(1-30)$ & $11 \cdot 8 \pm 0 \cdot 8(2-40)$ & $0 \cdot 56$ \\
GH $(\mu \mathrm{g} / \mathrm{l})$ & $15 \cdot 0 \pm 3 \cdot 6(0 \cdot 4-125)$ & $18 \cdot 7 \pm 4 \cdot 4(0 \cdot 2-374)$ & $0 \cdot 98$ \\
IGF-I $(\mu \mathrm{g} / \mathrm{l})$ & $616 \pm 46(65-1432)$ & $636 \pm 43(24-1976)$ & $0 \cdot 90$ \\
Active/Controlled & $41 / 12$ & $66 / 33$ & $0 \cdot 23$ \\
Eugonadal/Hypogonadal & $19 / 34$ & $7 \cdot 36 \pm 0 \cdot 98(1-23)$ & $0 \cdot 0005$ \\
Duration of hypogonadism (years) & $5 \cdot 5 \pm 0 \cdot 70(1-16)$ & $0 \cdot 19$ \\
\hline
\end{tabular}

Data are expressed as mean \pm SEM (range in parentheses). BMI, body mass index. 


\section{Discussion}

To the best of our knowledge, this is the largest study performed to evaluate the effects of acromegalic disease on BMD. Several previous studies have provided conflicting results when investigating small series of patients (Diamond et al., 1989; Ho et al., 1992; Ezzat et al., 1993; Kotzman et al., 1993; Kayath \& Vieira, 1997; Lesse et al., 1998; Longobardi et al., 1998; Chiodini I et al., 2001). This might be due to several factors. First, enrolment of patients with active or inactive disease, therefore with a wide range of GH and IGF-I levels. Second, in acromegaly, the deficiency of gonadal hormones due to the high prevalence of hypogonadism (Katznelson et al., 2001) is an important factor modulating bone metabolism. Finally, these modulating variables might differently influence bone tissue at LS and FN. In our opinion the conflicting results reported in previous papers could be due to the small samples studied and to the heterogeneity of different series (Diamond et al., 1989; Ho et al., 1992; Ezzat et al., 1993; Kotzman et al., 1993; Kayath \& Vieira, 1997; Lesse et al., 1998; Longobardi et al., 1998). For these reasons, we studied a large sample of acromegalics to evaluate the effect of GH excess on bone at different sites in relation to gender, gonadal status and disease activity. Our study suffers some limitations mainly due to its retrospective design: in particular, the different devices utilized and the availability of only areal BMD in a disease in which bone geometry could be altered. To overcome these limits, we expressed BMD data as Z-values in respect to control population of each centre; furthermore we also evaluated spinal trabecular volumetric bone mass in a subgroup of patients with active disease by QCT. The strong correlation of BMD measured by QCT and DXA, which is well known in normal subjects, suggests that in our active acromegalic patients data on areal BMD are informative of those on volumetric bone mass, at least in trabecular bone.

Our data show that at spine BMD was higher in eugonadal and lower in hypogonadal patients compared with controls. This finding demonstrates that at the spine the anabolic effect of GH becomes evident only in the presence of a normal gonadal status, whereas it is overridden by the catabolic effect of hypogonadism. This effect is independent of disease activity and gender. Our findings suggest therefore that conflicting results reported in the literature are likely to be partially due to a different prevalence of eu- and hypogonadal patients in their samples (Diamond et al., 1989; Ho et al., 1992; Ezzat et al., 1993; Kotzman et al., 1993; Kayath \& Vieira, 1997; Lesse et al., 1998; Longobardi et al., 1998).

BMD at FN was higher in acromegalic patients with active disease than in controls, independent of gonadal status. This finding suggests that at this site the anabolic effect of GH excess overcomes the catabolic effect of hypogonadism. Once again, no gender effect was observed. Our finding of different effects of hypogonadism, on either LS or FN in active acromegaly, is in agreement with recently reported data in an animal model (Morimoto et al., 2000). No difference was observed at FN between patients with controlled disease and reference population, suggesting that at this skeletal site the anabolic effect of previous chronic GH excess is reversible.

BMD at the FN did not correlate with 'duration of disease' and 'duration of hypogonadism', probably due to the crosssectional design of our study. The multiple regression analysis, however, showed that disease activity, as evaluated by IGF-I, significantly correlated with BMD measured at FN, a site composed prevalently of cortical bone. Our observation is in agreement with the previously observed association between BMD measured at forearm and serum IGF-I levels (Diamond et al., 1989), suggesting that the anabolic effect of chronic GH excess is more evident at cortical bone. Our data suggest that this effect is lost after normalization of IGF-I levels. BMD at spine correlated with 'duration of acromegaly' and 'duration of hypogonadism' and confirms that GH excess exerts its anabolic effect in relationship to gonadal status at a prevalently trabecular site such as LS. This effect is not lost after normalization of IGF-I levels. However, great caution is needed in this issue because in order to evaluate the impact of duration of the disease, duration of hypogonadism and disease activity on the bone, the crosssectional design of our study could be inappropriate, and a longitudinal study should be performed to address these issues.

In conclusion, our data indicate that the anabolic effect of $\mathrm{GH}$ excess on bone in acromegalic patients is: (i) gender-independent; (ii) evident at spine only in eugonadal status regardless for disease activity; and (iii) evident at femoral neck only in presence of active disease regardless for gonadal status.

\section{References}

Albright, F. \& Reifeinstein, E.C. (1948) The Parathyroid Glands and Metabolic Bone Disease: Selected Studies, pp. 188-196. Baltimore: Williams \& Wilkins.

Chiodini, I., Trischitta, V., Carnevale, V., Liuzzi, A. \& Scillitani, A. (2001) Bone mineral density in acromegaly: Does growth hormone excess protect against osteoporosis? Journal of Endocrinological Investigation, 24, 288-291.

Diamond, T., Nery, L. \& Posen, S. (1989) Spinal and peripheral bone mineral density in acromegaly: the effects of excess growth hormone and hypogonadism. Annals of International Medicine, 111, 567-573.

Ezzat, S., Melmed, S., Endres, D., Eyre, D.R. \& Singer, F.R. (1993) Biochemical assessment of bone formation and resorption in acromegaly. Journal of Clinical Endocrinology and Metabolism, 76, 1452-1457.

Giustina, A., Barkan, A., Casanueva, F.F., Cavagnini, F., Frohman, L., Ho, K., Veldhuis, J., Wass, J., Von Werder, K. \& Melmed, S. (2000) Criteria for cure of acromegaly: a consensus statement. Journal of Clinical Endocrinology and Metabolism, 85, 526-529.

Guglielmi, G., Giannatempo, G.M., Blunt, B.A., Grampp, S., Glüer, C.C., Cammisa, M. \& Genant, H.K. (1995) Spinal bone mineral density by quantitative $\mathrm{CT}$ in a normal Italian population. European Radiology, 5, 269-275. 
Ho, P.J., Fig, L.M., Barkan, A.L. \& Shapiro, B. (1992) Bone mineral density of the axial skeleton in acromegaly. Journal of Nuclear Medicine, 33, 1608-1612.

Kaji, H., Sugimoto, T., Nakaoka, D., Okimura, Y., Kaji, H., Abe, H. \& Chihara, K. (2001) Bone metabolism and body composition in Japanese patients with active acromegaly. Clinical Endocrinology, 55, 175-181.

Katznelson, L., Kleinberg, D., Lee Vance, M., Stravou, S., Pulaski, K.J., Schoenfeld, D.A., Hayden, D.L., Wright, M.E., Woodburn, C.J. \& Klibanski, A. (2001) Hypogonadism in patients with acromegaly: data from the multi-centre acromegaly registry pilot study. Clinical Endocrinology, 54, 183-188.

Kayath, M.J. \& Vieira, G.H. (1997) Osteopenia occurs in a minority of patients with acromegaly and is predominant in the spine. Osteoporosis International, 7, 226-230.

Kotzman, H., Bernecker, P., Hübsch, P., Pietschmann, P., Woloszczuk, W., Svoboda, T., Geyer, G. \& Luger, A. (1993) Bone mineral density and parameters of bone metabolism in patients with acromegaly. Journal of Bone and Mineral Research, 8, 459-465.

Lesse, G.P., Fraser, W.D., Farquharson, R., Hipkin, L. \& Vora, J.P. (1998) Gonadal status is an important determinant of bone density in acromegaly. Clinical Endocrinology, 48, 59-65.

Longobardi, S., Di Somma, C., Di Rella, F., Angelillo, N., Ferone, D.,
Colao, A., Merola, B. \& Lombardi, G. (1998) Bone mineral density and circulating cytokines in patients with acromegaly. Journal of Endocrinological Investigation, 21, 688-693.

Morimoto, I., Kai, K., Okada, Y., Okimoto, N., Uriu, K., Akino, K., Yamashita, S., Nakamura, T. \& Eto, S. (2000) Skeletal changes in rats bearing mammosomatotrophic pituitary tumors: a model of acromegaly with gonadal dysfunction. Bone, 26, 255-261.

Parazzini, F., Negri, E. \& La Vecchia, C. (1992) Reproductive and general lifestyle determinants of age at menopause. Maturitas, 15, 141-149.

Anonymous. (2001) Report of National Institute on Aging Advisory Panel on testosterone replacement in men. Journal of Clinical Endocrinology and Metabolism, 86, 4611-4614.

Scillitani, A., Chiodini, I., Carnevale, V., Giannatempo, G., Frusciante, V., Villella, M., Pileri, M., Guglielmi, G., Di Giorgio, A., Modoni, S., Fusilli, S., Di Cerbo, A. \& Liuzzi, A. (1997) Skeletal involvement in female acromegalic subjects: the effects of growth hormone excess in amenorrheal and menstruating patients. Journal of Bone and Mineral Research, 12, 1729-1736.

Seeman, E., Wahner, H.W., Offord, P., Kumar, R., Johnson, W.J. \& Riggs, B.L. (1982) Differential effects of endocrine dysfunction on the axial and the appendicular skeleton. Journal of Clinical Investigation, 69, 1302-1309. 